\title{
Subject Visit Start Day
}

National Cancer Institute

\section{Source}

National Cancer Institute. Subject Visit Start Day. NCI Thesaurus. Code C88001.

The day of the week a subject visit began. 